\title{
Ground Data are Essential for Biomass Remote Sensing Missions
}

Jérôme Chave ${ }^{1}$, Stuart J. Davies ${ }^{2}$, Oliver L. Phillips ${ }^{3}$, Simon L. Lewis ${ }^{3,8}$, Plinio Sist ${ }^{4}$, $\cdot$ Dmitry Schepaschenko $^{5}$, John Armston ${ }^{6}$, Tim R. Baker ${ }^{3}$, David Coomes ${ }^{7}$, Mathias Disney ${ }^{8,9}$, Laura Duncanson ${ }^{6}$, Bruno Hérault ${ }^{4,10}$, Nicolas Labrière ${ }^{1}$, Victoria Meyer ${ }^{11}$, Maxime Réjou-Méchain ${ }^{12}$, Klaus Scipa ${ }^{13}$, Sassan Saatchi ${ }^{11}$

\section{Abstract}

Several remote sensing missions will soon produce detailed carbon maps over all terrestrial ecosystems. These missions are dependent on accurate and representative in situ datasets for the training of their algorithms and product validation. However, long-term groundbased forest-monitoring systems are limited, especially in the tropics, and to be useful for validation, such ground-based observation systems need to be regularly revisited and maintained at least over the lifetime of the planned missions. Here we propose a strategy for a coordinated and global network of in situ data that would benefit biomass remote sensing missions. We propose to build upon existing networks of long-term tropical forest monitoring. To produce accurate ground-based biomass estimates, strict data quality must be guaranteed to users. It is more rewarding to invest ground resources at sites where there currently is assurance of a long-term commitment locally and where a core set of data is already available. We call these 'supersites'. Long-term funding for such an inter-agency endeavour remains an important challenge, and we here provide costing estimates to facilitate dialogue among stakeholders. One critical requirement is to ensure in situ data availability over the lifetime of remote sensing missions. To this end, consistent guidelines for supersite selection and management are proposed within the Forest Observation System, long-term funding should be assured, and principal investigators of the sites should be actively involved.

Keywords Biomass, Calibration, Forest, In situ data, Validation

\section{Introduction}

The global carbon cycle is being altered by anthropogenic activities: carbon dioxide and other economyrelated greenhouse gas emissions have steadily increased since the 1960s (Le Quéré et al. 2018). This has already had detectable consequences on the mean temperature of our planet (IPCC 2018). Land ecosystems hold a large potential for carbon storage. For example, it has been estimated that allowing Neotropical secondary forests to regenerate, without further human intervention, may enable Latin America and the Caribbean to be carbon-neutral for decades (Chazdon et al. 2016). Also, protecting intact forests is essential to ensuring carbon storage and many other ecosystem services (Pan et al. 2011). Thus, conserving existing intact forests, in combination with restoring and managing sustainably degraded forests, is almost certain to be a key action to help meet the Paris Accord targets. The idea of financially incentivizing local and national initiatives to spare forest land and favour reforestation has thus received further attention, as evidenced by the United Nations' Reduced Emissions from Deforestation and forest Degradation (REDD+) program.

The REDD+ framework is predicated on the ability to measure the differential amount of carbon stored in land ecosystems as a result of a change in policy compared to a defined business-as-usual scenario. This presupposes that instruments and methods are in place for monitoring, reporting and verification of land carbon budgets, yet there remain great challenges in this area (Grassi et al. 2017). In many temperate countries, which have largely built their political system around wood as a key commodity, elaborate systems of forest resource assessment and management were established early on, and they have been operated by national forest services. Thus, nationally determined carbon contributions are relatively reliable in the temperate zone, where forest biomass stocks are based on well established 
sample-based forest inventories (Fridman et al. 2014). However, the political history of many tropical or subtropical countries has been such that national forest inventory systems are either young or absent, in spite of efforts by the UN Food and Agriculture Organization (FAO) to set up such systems in several countries since the 1990s (Schimel et al. 2015). This situation is now changing, with national forest inventories being developed in Brazil and the Democratic Republic of Congo (Xu et al. 2017).

Remotely sensed approaches to estimate carbon stocks have emerged as a solution to this problem, and several missions are planned in the 2018-2022 period, including BIOMASS (P-band radar satellite by ESA), NISAR (L-band radar by NASA and ISRO), and GEDI (lidar onboard the ISS by NASA). These missions will not measure carbon stocks directly, but they will use proxies of forest structure, volume, and biomass components that correlate with the aboveground carbon stocks. Canopy height is measured by lidar and polarimetric interferometry, and tall forests tend to hold more carbon than shorter ones. The second physical quantity related to forest carbon store is the wood volume and water content which influence the backscattered electromagnetic energy measured at P-band $(\sim 70 \mathrm{~cm})$ or L-band $(\sim 25 \mathrm{~cm})$ wavelengths (Le Toan et al. 2011; Saatchi et al. 2011a, b; Shugart et al. 2010). Thus, these missions will collect data that can be empirically related to forest carbon content.

Because forest carbon stores are indirectly inferred from satellite sensors, with questionable assumptions about their dependence on forest structure and water content, it is essential that the planned missions make use of accurate ground estimates of carbon stocks to train their inversion algorithms and validate their products. However, estimating biomass on the ground is a challenge in itself and ecologists and foresters have struggled with this problem for a long time. Inevitably, providing inaccurate carbon stock estimates to the Earth Observation (EO) community will result in uncertain (and potentially biased) carbon maps, and this would have serious downstream effects on the usefulness of these maps in policy. For instance, even though pantropical biomass maps inferred from remote sensing have been available for some time now (Saatchi et al. 2011a, b; Baccini et al. 2012), the UN Intergovernmental Panel on Climate Change (IPCC) has been reluctant to recommend their widespread use over national inventories because of possible calibration issues. Here, we offer a perspective from the ground up and propose a strategy for gathering reliable ground-based measurements and biomass estimates that will be useful to the various Earth Observation missions aimed at quantifying forest structure and carbon stock at a global scale.

Overarching principles are summarized here, and echo meetings jointly held on ground data and upcoming land Earth Observation missions (NASA-ESA-Smithsonian Workshop 2016; ISSI ESA Meeting, Bern, 2017). First, the focus of all of these missions is primarily tropical. Many forested extratropical countries already have a forest inventory assessment in operation. In contrast, ground-based monitoring systems are sorely lacking in the tropics (Schimel et al. 2015). Forest extent in the tropics is still very substantial, by far the most living biomass is located in the tropics (63\% of carbon in intact tropical forests, against $15 \%$ in boreal forests and $13 \%$ in temperate forests, according to a comprehensive estimate, Pan et al. 2011). Second, in order to map change in forest ecosystems, ground sites need to be regularly revisited (Frolking et al. 2009). It is impossible financially and logistically to maintain thousands of sites without long-lasting governmental or international support. These observation systems need to be maintained at least over the lifetime of the planned missions, but it is likely that they will find even greater value if made permanent through binding agreements-here we provide costing estimates to facilitate the discussion of this question, while acknowledging that informed recommendations for the calibration and validation of the missions are dependent on the nature of the algorithm, on the resolution of the data, and on the mission duration, and are therefore beyond the scope of the present study. Third, estimating biomass correctly in situ remains a delicate business, and strict data quality control must be guaranteed to users. 


\section{Principles of Ground-Based Biomass Estimation}

Aboveground biomass (AGB) is the total amount of dry matter of live trees held aboveground in a plot. It is a key parameter for a range of applications, including greenhouse gas accounting, forest fire assessment, management of the timber industry, monitoring of landuse change, and ecosystem science. Currently, accurate AGB estimates can be obtained only by labour-intensive fieldwork (plot inventories) conducted by trained operators. The AGB of each tree is estimated from measured variables using an allometric model $A G B=f(\rho, D, H)$, where $\rho$ is the stem wood density of the focal tree, $D$ its trunk diameter, and $\mathrm{H}$ its total height. Trunk diameter is usually measured at breast height $(130 \mathrm{~cm}$ aboveground) in forests, but in non-forest habitats, trees tend to branch low, and standard measurements are lower, e.g. at $10 \mathrm{~cm}$ aboveground in Australia (Paul et al. 2016). For the largest trees, diameter needs to be measured above buttresses (Sillett et al. 2019). Such allometric models are constructed from destructively harvested trees in which AGB and the other variables are all measured directly. Oven-dry biomass is approximately $47 \%$ carbon, the conversion from AGB to aboveground carbon stores is easy, and the two notions often used interchangeably. Importantly, a thorough and recent analysis of wood carbon content showed that carbon content varies predictably among plant functional types and ranges from 41 to $51 \%$, and this source of variation should be carefully considered (Martin et al. 2018). Since measuring wood density for each tree would be too labour-intensive, recent studies have used species-mean wood density values, taken from publicly available lookup tables. It follows that the reliable taxonomic identification of each tree is essential for an accurate estimate of its AGB.

The tree-level AGB estimates are then summed across trees and over the plot, to produce an aboveground biomass value at the plot level, usually expressed in $\mathrm{Mg} / \mathrm{ha}$ of dry matter. AGB is not measured in forests, but estimated (Clark and Kellner 2012), and errors due to the estimation of height, wood density, and choice of the allometric model usually result in a plot-based AGB uncertainty that is non-negligible. AGB is estimated to within $50 \%$ absolute error for a single tree (Chave et al. 2014). This absolute error, when propagated at stand level, is around $10 \%$ at the 1-ha scale (Réjou-Méchain et al. 2014).

In most current applications, live below ground biomass is usually inferred from AGB using standard root-shoot ratios (Mokany et al. 2006; Paul et al. 2019). Although this poses important and specific challenges, the issue of estimating biomass components other than AGB (belowground biomass, soil carbon, coarse woody debris) is not covered in this contribution.

In the past few decades, estimation of carbon stocks and AGB in the tropics has been based on permanent forest plots with measurements of tree trunk diameters, and often tree mapping and species identification of the trees. AGB can then be estimated per tree and then summed over all trees in a stand (Brown and Lugo 1982). Permanent sampling forest plots are thus the basic unit of biomass measurement. Their size ranges from 0.01 ha $(10 \times 10 \mathrm{~m})$ to almost 100 ha $(1000 \times 1000 \mathrm{~m})$. Within these plots, all trees above a given trunk diameter threshold (usually $10 \mathrm{~cm}$ ) are counted in the census. These trees are mapped (usually to the nearest $m$ or ideally better-but unfortunately quite often much less accurately), they are marked permanently with a tag, and their trunk diameter is measured at a standard position on the trunk (usually $130 \mathrm{~cm}$ aboveground, or $50 \mathrm{~cm}$ above buttresses or irregularities, if present). The point of measurement is noted by a permanent paint mark. A sample of trees may also have their height dimension measured, and as far as possible, all trees are identified taxonomically. The numbers of trees $\geq 10 \mathrm{~cm}$ in one hectare of mature moist forest varies from 300 to 1000. The number of tree species per hectare is up to 30 in temperate forests and up to 300 in the tropics (Gentry 1988). Thus, species identification can be a considerable challenge. 
Temperate and boreal forests present different challenges than tropical forests, and several temperate countries can rely on National Forest Inventories (NFIs) constructed using statistical sampling theory. These are designed to provide inferences of biomass and other commodity values at regional or jurisdictional levels using a large number of small plots (Smith 2002). These plots can be used to validate the Earth Observation biomass products, but their use for algorithm development and training is more limited due to plot size and potentially large plot-level uncertainty of ground biomass. The challenge of mobilizing temperate-country ground data is an important one but, because most of the world's high biomass forests are in the tropics, we here emphasize the tropical zone. We also note that vast regions like extratropical Asia are missing NFIs, and it would be important to better account for in situ forest information in these regions.

Aerial lidar scanning (ALS) has been intensively used for estimating tropical forest biomass (Drake et al. 2002; Asner et al. 2010), and the literature suggests that, if ALS data can be calibrated locally with permanent sampling plots, the resulting biomass maps are unbiased and reliable. (They have a relative uncertainty of $<20 \%$ at the 1-ha scale.) Establishing high-resolution biomass maps at 1000-ha (10 km2) scale would result in a 100-fold increase over plot data, and the 1000-ha scale is typically the area surveyed around permanent field stations in the tropics: this means that the sites are within walking distance and the ALS-derived biomass map can be thoroughly ground-truthed. However, since forests are constantly changing, algorithm training and validation of ALS data is impossible without nearcontemporaneous fieldwork.

\section{High-Quality Carbon Estimates Require Long-Term Study Sites}

\subsection{Forest Dynamics and Tree Inventories}

Collections of small plots can offer a representative sample of the landscape-scale variability of biomass, but lack the temporal dimension that is also critically important for understanding the system. Forest changes include (1) secular changes in mature forests driven externally by climate (e.g., increasing/declining growth rate), (2) sudden, stochastic changes (e.g., drought-, flood-, wind-, pest-, fire-induced mortality), (3) successional development (e.g., savanna thickening into forest, floodplain forests). Anthropogenic impacts on forests are equally important, complex, and ubiquitous. As a result, it is not enough to use forest biomass estimates at a given point in time, but forests should be measured repeatedly in situ. Most existing permanent census plots are recensused by trained teams of foresters and botanists every $4-5$ years, funds permitting, a monitoring revisit frequency that is sufficient if tree turnover rates are around 1-3\%/year.

Another reason for measuring forest stands repeatedly is that some measurement errors may affect biomass estimates far more than others. A small number of large trees holds a large fraction of the biomass in a stand, and these are the most difficult to measure in the field. Assume a $100 \times 100 \mathrm{~m}$ stand of tropical forest contains around 500 trees of trunk diameter $>10 \mathrm{~cm}$, and the oven-dry aboveground biomass is 300 tons or more, a typical situation in moist tropical forests. Thus, on average, a tree weighs 0.6 ton. However, the distribution of tree weights is hugely skewed, since according to one study conducted in intact tropical forests, $41 \%$ of the aboveground biomass was held in trees above $60 \mathrm{~cm}$ in trunk diameter (Lutz et al. 2018). In tropical forests, historical permanent plots were often established by botanists to explore plant diversity. In these cases, initially, little attention may have been paid to carefully measuring the largest trees, and plots were often located based on convenience more than based on a sampling protocol (plots installed by foresters tended to have the opposite focus: large trees measured well, but little attention paid to the botany of non-commercial species). Clark and Clark (2000) provided the first comprehensive study comparing different carbon sampling strategies in the tropical forest of La Selva, Costa Rica. They showed that measuring trunk diameter above buttresses was key to a proper estimate of AGB (Fig. 1; see also Condit 1998). 
It is essential to realize that, for many sites, the history of the plots is complex, and data quality may have changed over time. Therefore, the issue is not only to process pre-existing data, but also to critically appraise the field collection protocols to ensure that legacy data are made available and associated with an uncertainty assessment that accounts for the history of data acquisition, that varies greatly from site to site and among groups of data collectors.

In the tropics, a major contribution is required from developing country scientists and technicians. The ground data they produce are hard-won and need to be repeated at regular intervals. The participants in our plot networks span hundreds of tropical forested localities across up to 50 different nations. Such work involves obvious logistical complexities of organizing ground data collection, institutional collaborations, intellectual property, permits, and health and safety protocols to allow remote fieldwork and plant collection across so many countries while complying with protected area regulations. In addition, a key challenge is to harmonize datasets and differing existing ground biomass protocols. Consequently, because of this major effort and the heavy dependence on often specialized human labour, local researchers who lead collection campaigns and own ground-based measurements must be involved as scientific collaborators, and field teams adequately trained, equipped, insured, and paid.
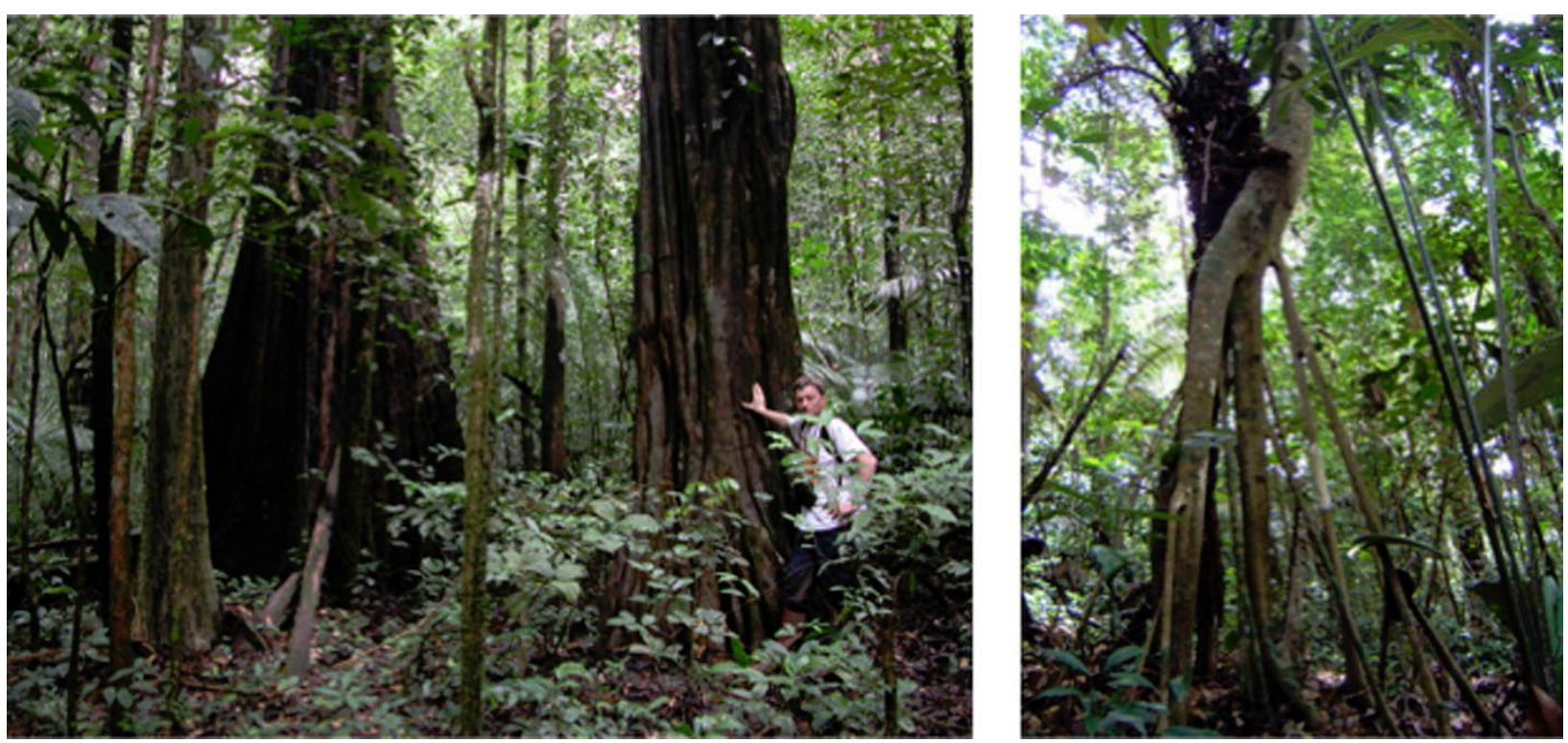

Fig. 1 Fig. 1 Permanent plot in intact lowland tropical forest at the Nouragues station, French Guiana. Left: Large, buttressed, trees encompass the majority of the aboveground biomass stock. Right: A pioneer tree (Cecropia sciadophylla Mart.) has grown from the top of a palm, causing issues of trunk diameter measurement. Situations like this one are resolved only with proper field protocols

\subsection{Calibration and Validation Strategies for Earth Observation Missions}

The aboveground biomass data from in situ plot networks are an essential requirement of current EO missions. Several biomass EO missions are currently in the process of developing their algorithms or preparing their validation plans utilising this type of ground data. This includes the Global Ecosystem Dynamics Investigation (GEDI) mission, launched in December 2018 (NASA), the NISAR mission (NASAISRO synthetic aperture radar, launch in 2021), and the BIOMASS mission (ESA, launch in 2022). The ground data already collected as part of these efforts are remarkably similar, even if the requirements

differ slightly.

The major requirement is that ground biomass values be available, based on intensive tree inventories, and reliable biomass estimation methods. EO missions have included requirements about quality assessment of these plot-based biomass estimates, because improperly estimated ground biomass 
values are not rare, and failure to account for unreliable data will result in serious problems in the calibration and validation plan. The GEDI, NISAR and BIOMASS missions have each complied and collected data from plot networks, and have recently shared the metadata of their efforts. The GEDI science team, the most advanced to the date of the submission of this contribution, has assembled a dataset of 105 sites. These data were contributed by a variety of projects and are thus in-kind contributions. They span the major biomes and represent almost 1400 ha of surveyed plots, of which $40 \%$ are in the Neotropics (557 ha), 12\% in Africa (173 ha), and 7\% in tropical Asia (108 ha). The NISAR mission cal/val team has assembled data for 77 sites, with quite some overlap with that of the GEDI science team. BIOMASS is the least advanced, including 6 sites, two in French Guiana (Neotropics) and four in Gabon, Africa (Labrière et al. 2018), and a total sampled area of 227 ha. In addition to permanent plot data, all three missions include airborne lidar scanning (ALS) in their ground dataset. ALS has been shown to be a critically component to EO missions, because it provides invaluable information on forest structure, in a carefully georeferenced format, and this can be used to upscale plot-based biomass estimates to landscape-scale biomass maps.

In comparison with these datasets, forest plot networks include far more information (Table 1). For instance, the Smithsonian Institute's ForestGEO coordinates 245 ha of forest across 4 sites in Africa, and over 250 ha in tropical Asia (Anderson-Teixeira et al. 2015). The Rainfor network in South America, AfriTRON in tropical Africa and T-Forces in Asia, managed by University of Leeds, coordinates no less than 400 ha of plots in Amazonia alone (Mitchard et al. 2014) and 315 ha in tropical Africa (Lewis et al. 2013). These latter three networks are all stored in ForestPlots.net allowing quality control and data processing chains to be applied across all three networks. Among these various networks, there is almost no overlap, and they do not include independent large projects such as forest management experiments. These have recently been coordinated by the Tropical managed Forest Observatory (TmFO), with almost 1200 ha of forests permanently monitored (Sist et al. 2015). Also, a network of secondary forest plots has been established in the Neotropics and coordinates effort on forest regeneration (Chazdon et al. 2016). Our estimate is that the area of tropical forests that are currently monitored globally is in excess of 2500 ha by these four networks and not accounting for many more projects. This, however, remains a minuscule fraction of the total area covered by forest worldwide, and the biomass estimation challenge is therefore one of upscaling.

\subsection{Supersites}

Based on our knowledge of available data within the partners, it would be more cost-effective to prioritize a limited number of ecologically representative sites around the world. We call this the 'supersites' concept. Such sites combine intensive and long-term fieldwork data, airborne vegetation monitoring, and ancillary information, such that reliable landscape-scale biomass estimation is possible (Fig. 2). This idea of establishing long-term sampling sites with EO applications in mind is fundamentally the same as that of the US long-term ecological research sites (LTER) in place since the 1970s, and the International Biological Program (Golley 1993). The Committee on Earth Observing Satellites (CEOS) Working Group on Land Product Validation has officially endorsed a supersite concept not only for biomass, but to identify and promote the collection of validation data for the wide range of Essential Climate Variables products that are currently available or expected in the coming years.

Meeting high data quality in the tropics is possible at or near research stations with existing infrastructure and resources, and with the potential to upgrade datasets, funds permitting. Based on previous experience with the TropiSAR campaign (French Guiana; Dubois-Fernandez et al. 2012), and AfriSAR mission (Gabon), we propose that these supersites be selected based on the following specific requirements: (1) availability of at least 10 already established 1-ha permanent sampling plots, ideally well-distributed across the landscape, capturing local gradients of biomass. The plots should be established according the best tropical forestry standards (see RAINFOR or Center for Tropical Forest 
Science protocols, e.g., Condit 1998); (2) availability of tree height measurements at each of these plots (for all trees or at least a representative sample of trees); (3) availability or potential future collection of ALS coverage over at least 1000 ha, flown over the permanent plots, with minimal quality requirements (i.e. such that 1-m canopy elevation models can be constructed); (4) availability of a weather station and, optionally, automated soil moisture monitoring (ideally encompassing the landscape-scale variation of soil moisture). Inasmuch as possible, these data should be acquired at the same time.

Table 1 List of characteristics for some of the international tropical forest-monitoring networks currently in operation

\begin{tabular}{|l|l|l|l|l|l|l|l|l|}
\hline Network & Countries & Plots & $\begin{array}{l}\text { Plot } \\
\text { sample } \\
\text { area (ha) }\end{array}$ & Trees & $\begin{array}{l}\text { Specie } \\
\mathrm{s}\end{array}$ & $\begin{array}{l}\text { Measure- } \\
\text { ments }\end{array}$ & $\begin{array}{l}\text { Forest } \\
\text { types }\end{array}$ & $\begin{array}{l}\text { Regional } \\
\text { focus }\end{array}$ \\
\hline ForestGEO & 26 & 65 & $16-120$ & $\begin{array}{l}6.5 \\
\text { million }\end{array}$ & $12^{\prime} 000$ & 20 million & Primary & Global \\
\hline RAINFOR & 9 & 400 & $\begin{array}{l}0.2-9, \\
\text { mostly } 1\end{array}$ & $280^{\prime} 000$ & $5^{\prime} 500$ & 2 million & Primary & $\begin{array}{l}\text { South } \\
\text { America }\end{array}$ \\
\hline AfriTRON & 11 & 320 & $\begin{array}{l}0.2-10, \\
\text { mostly } 1\end{array}$ & $170^{\prime} 000$ & $1^{\prime} 800$ & $600^{\prime} 000$ & Primary & Africa \\
\hline TmFO & 10 & 517 & $\begin{array}{l}0.25-27, \\
\text { mostly 1 }\end{array}$ & $\begin{array}{l}300^{\prime} 000- \\
400^{\prime} 000\end{array}$ & - & 6 million & Logged & $\begin{array}{l}\text { Pan- } \\
\text { tropical }\end{array}$ \\
\hline
\end{tabular}

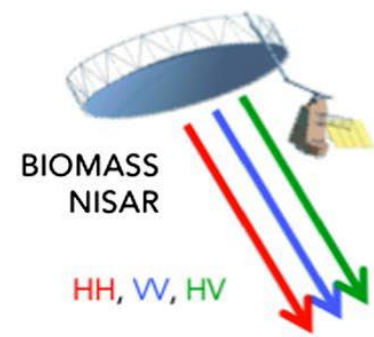

GEDI

\section{Airborne lidar scanning survey (ca 1000 ha)}

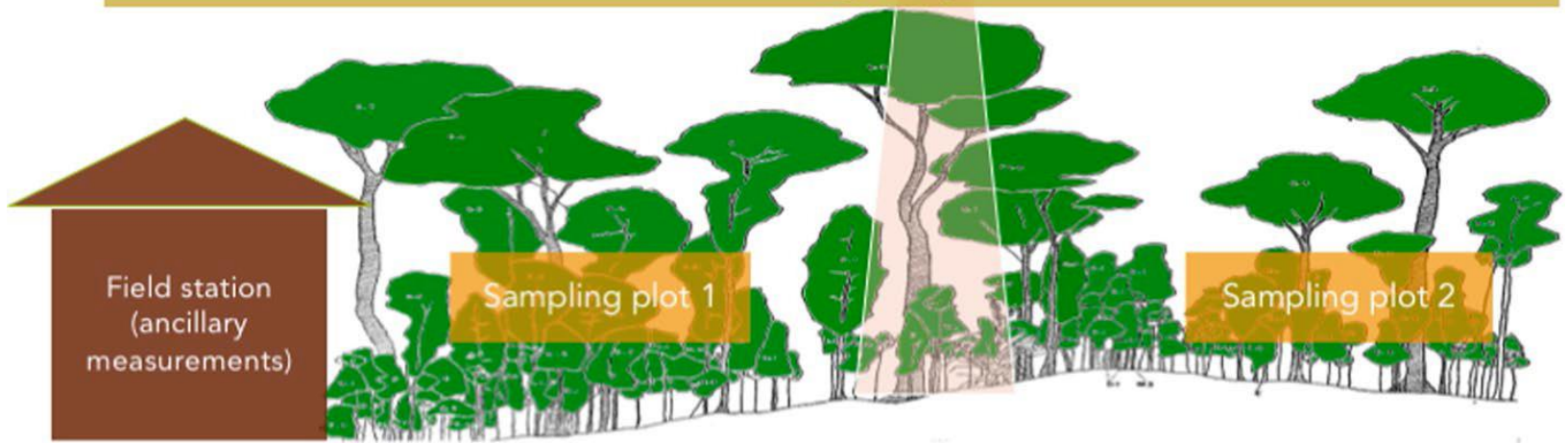

Fig. 2 The supersite concept. Relatively few sites with long-term investment by plot principal investigators, and the potential to upgrade the sites. The background is taken from Ashton (1964, Kuala Belalong, Brunei)

We also propose to implement terrestrial lidar scanning (TLS) surveys of the permanent plots. TLS surveys are no substitute for forest tree inventories, but they have the potential to complement them usefully: they provide an accurate measure of individual tree volume, a measure of total tree height, and an accurate correction of stem geolocation (relative, at stand scale). They also give access to the details of forest structure that may be important in modelling canopy reflectance at these sites (Calders et al. 2018). This considerably increases the quantity and quality of much of the key parameters on which all of the other estimates rely. The resulting tree volume data can also be used to augment existing allometric relationships used to generate tree biomass estimates, particularly across a much 
larger range of tree size and including many more large trees (Disney et al. 2018). Tree volume and tree mass may differ significantly in the case of hollow trunks, and large trees tend to be more often hollow than small ones (Nogueira et al. 2006). Recently, drone-based alternatives for terrestrial lidar scanning have been proposed and they present the additional advantages of scanning the canopy tops and of producing already-stitched point clouds, over large areas typically several hectares (Brede et al. 2017). While this technology still requires development, it would be important to explore its applicability to the establishment and monitoring of forest supersites.

\subsection{Drivers of Biomass Stocks and Geographical Coverage}

Forest structure varies at all spatial scales. Determining the optimal sampling strategy for Earth's forests requires research into the drivers of biomass stocks, which in turn depends on spatially explicit maps of forest structure that will not be available until the new mission products come online. It is difficult to segment tropical forests worldwide into forest types that would both make ecological sense and would be optimal for the training of biomass retrieval algorithms. For instance, two forests may have a similar structure, yet display species with different wood densities, resulting in very different biomass estimates. Also, the forest lower canopy may play a significant role in the radar backscattering properties, and, like wood density, this is not readily assessed remotely.

We therefore provide ecologically informed guiding principles for the selection of sites. Tropical forests vary in their structure and floristic composition, and this in turn impacts their biomass storage capacity (Malhi et al. 2004; Stegen et al. 2011). The four main driving factors of this variation are soil fertility, moisture supply, elevation, and disturbance regime. Therefore, forests often hold less biomass on very infertile or very fertile soils (Castilho et al. 2006). Also, dry tropical forests have less biomass, but there is also potentially a hump-shaped distribution of biomass with respect to annual precipitation whereby ever-wet forests tend to have lower biomass stocks than moist forests (Brown and Lugo 1982). Elevation is another important factor, and biomass usually declines with increasing altitude, although some exceptions exist, for example when trees of the oak family are present (Phillips et al. 2016). Finally, disturbed forests have a lower biomass than undisturbed ones. The foremost cause of disturbance in the tropics is anthropic, but other causes exist including wildfires, wind storms, insect predation, or diseases, and the frequency and intensity of natural disturbance exerts a critical control on intact forest wood density and biomass (Keeling and Phillips 2007; Johnson et al. 2016).

In addition, tropical forests have almost zero floristic overlap between the Neotropics (South America), Africa, Asia, and Oceania (including Papua New Guinea and Australia) with each biogeographic region having thousands of tree species whose architecture and unique identity helps to determine forest structure and biomass in that region. Assuming that three conditions (low, medium, high) are selected for each of the four major gradients in each of the four continents, the number of possibilities is $4 \times 34=$ 324.

Practically, when selecting sites for algorithm training or product validation, it is essential to include the full range of variability in biomass, i.e. high-biomass forests, typically moist tropical forests with biomass stocks more than 300-400 tons/ha, and up to 600 tons/ha, but also low-biomass forests, typically < 100-200 tons/ha (Sullivan et al. 2017). For instance, a relatively young secondary forest of ca. 20 years regrowing from clear-cutting holds about 100 tons/ha in tropical areas (assuming an accumulation rate of 5 tons/ha/year). Also, woodlands store 30-150 tons/ha. It would be important to include both secondary vegetation in the study landscapes, and to select dry vegetation types. These vegetation types are particularly important for the NISAR mission, which aims at estimating biomass up to 100 $\mathrm{Mg} / \mathrm{ha}$, above which L-band backscatter signals saturate with respect to biomass (Chapman and Kasischke 2018). 


\section{Building on Long-Term Forest Plots}

\subsection{The Forest Observation System}

Permanent plots provide the most accurate method for forest biomass estimation, which not only depends on biometric variables, but also on species-dependent estimates of wood density. Many sites across the forested tropics have on the order of ten 1-ha plots, because this sampling intensity is manageable. Much larger sampling intensities do exist, but they are rare. Further, while plots are often established randomly with respect to small-scale disturbance events, they are rarely formally randomised within strata across the landscape. Plots are often located to monitor specific environments (e.g. old-growth forest), or test hypotheses, and were not established to estimate biomass stocks at the landscape scale, as required by the various EO missions.

The European Space Agency has funded the Forest Observation System (FOS) as an effort to coordinate in situ activities in relation with the BIOMASS mission. The FOS includes several large international consortia who are addressing the issues of ground data sharing and standardization: RAINFOR, AfriTRON, and T-FORCES (led from the University of Leeds), ForestGEO (including CTFS; Smithsonian Institution). These consortia have a solid record in tackling key scientific questions, in engaging a community of collaborators and in standardizing forestry data. For up to 40 years now, they have been devoted to coordinating long-term research with permanent sampling forest plots. They have (1) established permanent sampling plots in tropical and temperate forests, (2) encouraged and carried out extensive plant collection and identification, (3) proposed robust protocols for accurate tree mapping, and measurement, (4) monitored existing plots repeatedly, and (5) established databases with a special emphasis on data quality control at the tree level, and have successfully incorporated historical databases.

Two additional networks of permanent forest plots have now been invited to join the Forest Observation System: TmFO (Tropical managed Forest Observatory; Sist et al. 2015) and AusCover (CSIRO). We are aware that many more groups of scientists and networks of plots have been established, but when examining inclusion of new sites into the FOS, it is essential to consider upstream quality assessment. It is preferable to build upon projects that have already established a data sharing policy, quality assessment procedures, and instruments for communication with principal investigators at each of the sites.

NASA and ESA are also in the process of establishing a Multi-mission Analysis and Algorithm platform (MAAP), which will house field plot, airborne lidar, and spaceborne datasets, including data from NISAR, GEDI, and BIOMASS. This will be a virtual open and collaborative environment, bringing together data, cloud-based computing resources, and collaborative tools. It will establish a collaboration framework between ESA and NASA to share data, science algorithms and computing resources in order to foster and accelerate scientific research conducted by NASA and ESA scientists. We intend that the Forest Observation System (FOS) will become an integral part of this multi-mission analysis platform, facilitating provision of field plot data such as from existing plots and new supersite data acquisitions.

Gathering calibration and validation data relevant to biomass for the Earth Observation community faces a number of challenges, and the FOS aims to address the most important ones. We here list the priorities: (1) ensuring the respect of intellectual property rights, (2) providing site principal investigators with a knowledge of the scientific challenges undertaken with their data, and (3) ensuring that datasets included in FOS are of the highest possible quality and are representative of all forest ecosystems.

A key aspect of the collaboration is that the intellectual property of the primary data remains with the site's principal investigator. This principle is upheld in the FOS data sharing policy (under preparation). Official data sharing policies are found, for instance for the Smithsonian Institute (white paper 'Sharing 
Smithsonian Digital Scientific Research Data from Biology', March 2011), for the RAINFOR project (white paper 'Ethical Code, Data Sharing \& Publication Policy for RAINFOR Participants', June 2009), and in TmFO's Memorandum of Understanding. Within the FOS, plot consortia are acting on behalf of the site principal investigators. Importantly, data providers are not asked to provide their primary (tree-by-tree) data. The data shared in the FOS are stand-level descriptors, including aboveground biomass estimates, that are obtained from a standardized procedure.

One of the most frequent complaints voiced by site principal investigators is that the data they are providing serve projects downstream of which they are not aware. This is to a large extent a communication problem, and one that can be solved through constant interaction with site principal investigators through a mailing list. Allied to this, sometimes data is used by scientists who make implausible scientific interpretations, without knowing the site, its history and other details. Some interaction with data providers often improves papers and quickens the pace of scientific discovery.

\subsection{Plot Data Requirements}

Minimal data requirements are here discussed. These data should be produced by the partners and provided to the Forest Observation System database.

A minimum set of site descriptors are included in the metadata. These include: (a) the name and contact (email) of the plot principal investigator(s); they should agree to be mentioned in the database (for privacy protection, this information is made available online in the password-protected part of the database); (b) the name of the partner institution(s) and individual in charge of data management; (c) the names of the funding bodies; and (d) some characteristic photographs of the forest.

The following plot information is important: (a) plot coordinates, which should be checked for the geodetic system and be provided in World Geodetic Survey WGS84; GPS coordinates should be of high accuracy, typically to within $10 \mathrm{~m}$ (but ideally with surveying GPS to within $\mathrm{cm}$ ), so as to facilitate coregistration with other datasets (ALS, TLS, and EO); plot coordinates should ideally refer to the centre and the four corners of the plot; (b) collection date and periodicity; number and date of censuses carried out should also be known; the census number for which AGB data are provided should be given; (c) the total sampled area, i.e. the horizontal projection of the on-ground sampled area (i.e. topography effects are ignored), and plot geometry; most plots are squares or rectangles; (d) the dataset should also document the relief (slope, exposition); in situations where aerial LiDAR is available, this usually provides accurate measurements of ground relief; (e) forest type (i.e. wet, moist, dry forests) and successional status should be documented. Note that networks have already faced the issue of postfield data standardization/filtering. However, it is not established that they all have settled to a common practice in all instances.

We also report on metadata for the tree inventory itself: (a) the number of trees $\geq 10 \mathrm{~cm}$ in trunk diameter; note that trees $<10 \mathrm{~cm}$ and other life forms are usually excluded in AGB estimates in case their contribution to $A G B$ is $<5 \%$; (b) a quality assessment index, reporting on whether points of measurement have been properly recorded for each tree; (c) an index reporting on the quality of taxonomic identification will also be needed; as a rough measure, the proportion of trees identified to species level, genus level, and family level is reported. In tropical forests, identification of $<50 \%$ of the trees to species level is far from unusual. Careful botanical identification by botany experts typically results in identification rates of $>90 \%$ of the trees, but may entail climbing trees to collect and significant downstream identification effort with botanists and herbaria; (d) plot-averaged wood density is the basal-area-weighted wood density of the trees in a plot. For plots with reliable taxonomic identifications, this may be deduced from census data and species-average wood density values; (e) mean canopy height of the plot, as inferred from direct tree height measurements or from airborne 
LiDAR measurements; if necessary, several canopy height metrics should be provided; quality-control metric: height of the largest measured tree, trunk diameter of the largest measured tree.

Finally, aboveground biomass and confidence intervals are computed and provided the plot scale, following an agreed single methodology across partners; the methodology will be made accessible for each database release, and data users should be prepared to changes in the methodology. An efficient strategy is to jointly develop a statistical routine such that several database formats can be accommodated and that perform the tasks of calculating biomass and canopy height at each site. The R statistical software is recommended because it is free, already widely used in the ecological research community, and networks such as ForestGEO or ForestPlots already have developed $\mathrm{R}$ routines for parsing the datasets and performing quality checks. We have established a package called BIOMASS that calculates biomass values and propagates uncertainty from tree measurement to stand-level estimates (Réjou-Méchain et al. 2017). This package is flexible and makes it possible to use user-supplied conditions or allometric equations.

\subsection{Candidate Supersites and Their Coverage of Environmental Gradients}

There are around 50-100 supersites already potentially available worldwide, and here, we discuss a list of 78 sites included as priority sites by the ESA-NASA cross-mission working group. All sites share a number of basic features including a long-term presence of scientists, existing forest monitoring programs, and willingness to collaborate in international scientific projects on the part of the principal investigators.

Taken together, these sites encompass much of the variability in forest types, and within each 1000-ha region of interest, these sites display a large spectrum in biomass ranges and disturbance histories. Figure 3 illustrates the location of sites that could be prioritized as supersites. A majority of the supersites are located in the tropics, reflecting the more pressing need for data in tropical forest environments. However, several sites were also selected outside of the tropical belt.

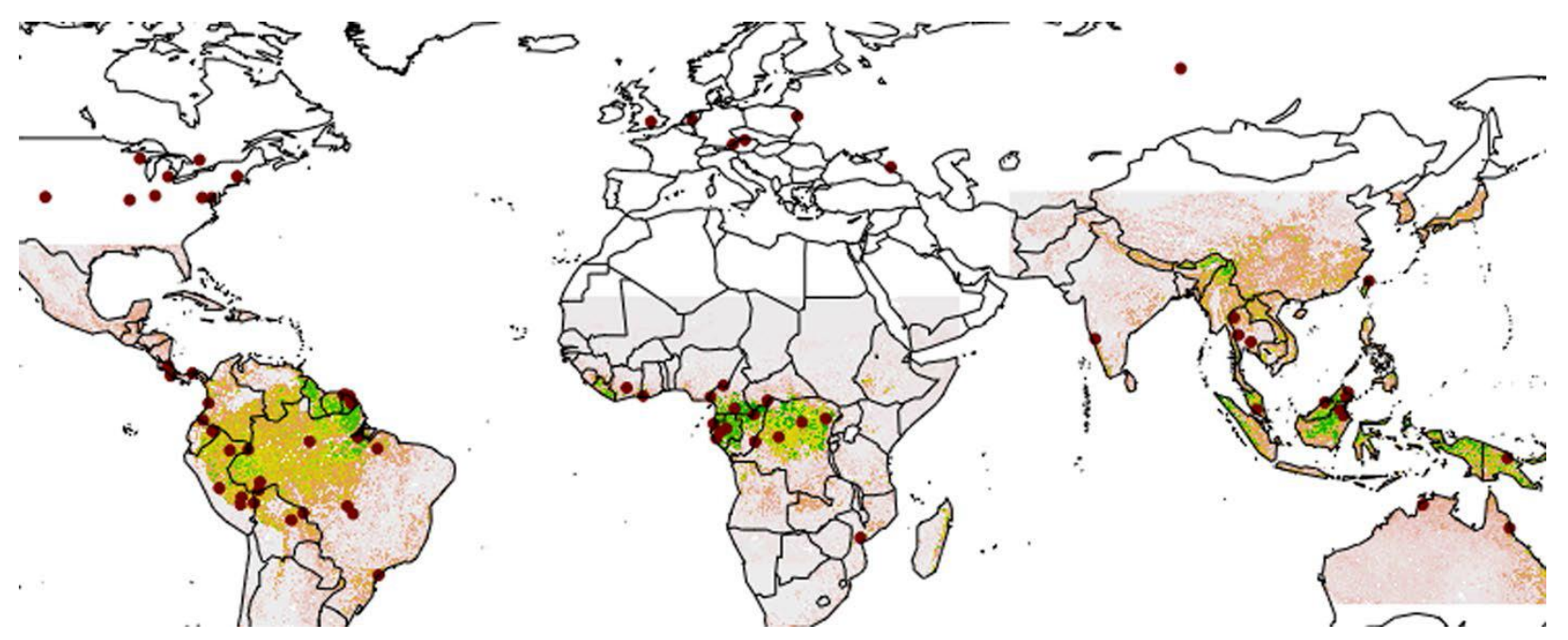

Fig. 3 Potential location of 78 candidate supersites. Proposed sites were selected to maximize geographical coverage, environmental and forest structure conditions, and logistical constraints of maintaining long-term sites. The background is Avitabile et al. (2016) carbon stock map

We also illustrate the coverage of these sites in terms of biomes and bioclimatic conditions (Fig. 4). The 78 sites currently being considered for the network of supersites span broad bioclimatic conditions, and although they are mostly located below $1000 \mathrm{~m}$ in elevation, a few sites $(n=6)$ are above this limit. As seen in Fig. 4, the current list of supersites does not include many dry forests, semi-deciduous tropical forests, or boreal forests. Also, warm temperate forests are currently under-sampled in this dataset. Finally, a large proportion of the sites are currently located in areas with less than $1 \%$ disturbance from 
2000 to 2017 (28 out of 78) but some are in highly disturbed landscapes. One example is the STREK site in Indonesia (TmFO), in which over $60 \%$ of the surrounding landscape has been deforested since 2000 , another example being the Pasoh plot (ForestGEO) with over 40\% of deforestation since 2000.

(a)

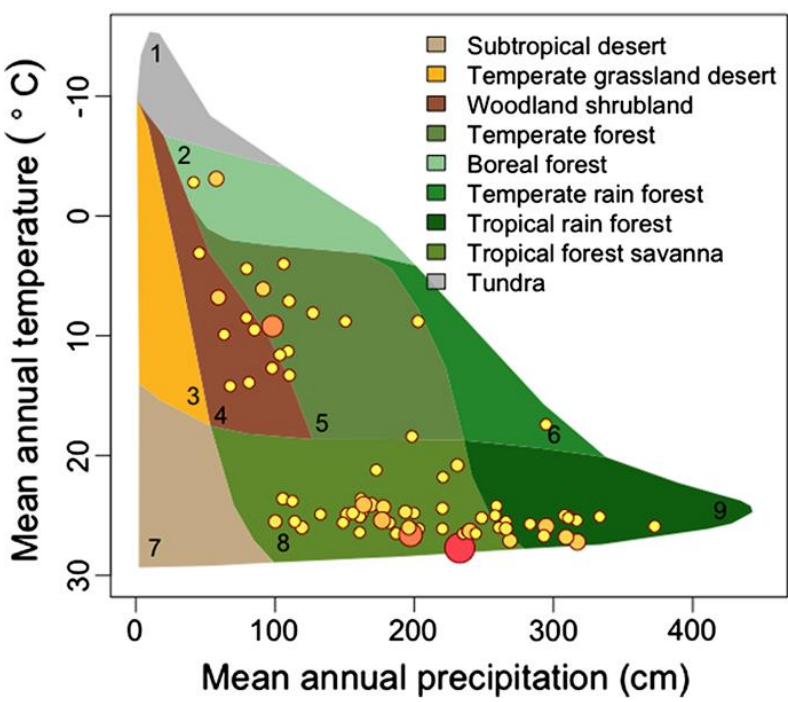

(b)

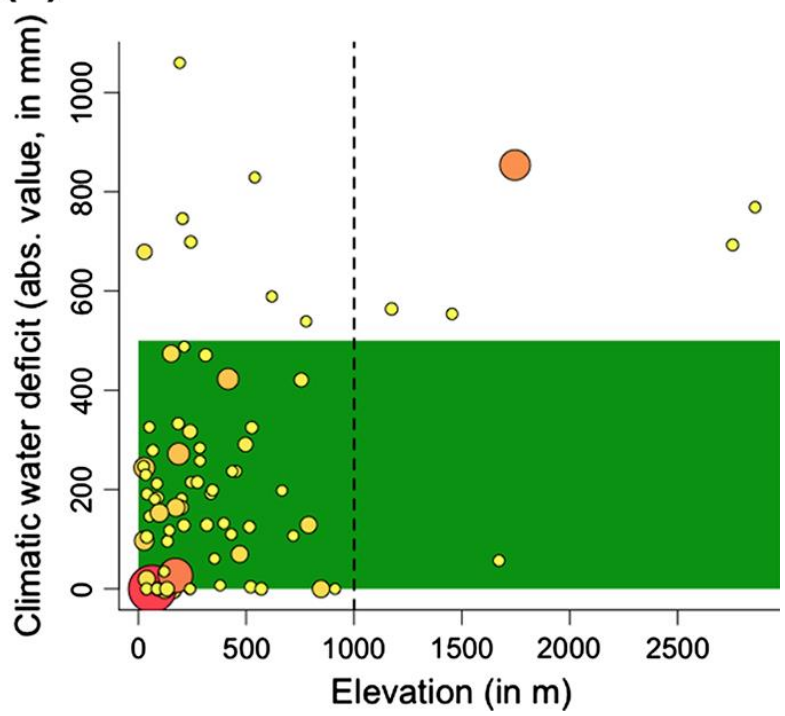

Fig. 4 a Environmental coverage of the 78 candidate supersites in bioclimatic space (Whittaker diagram). b Distribution of the candidate supersites across the range of elevation (in $m$ above sea level); drought stress, as measured by the climate water deficit: larger values represent more stressed environments (sites above 500 are usually ascribed to xeric habitats). In both panels, disturbance intensity is displayed with large red dots representing highly disturbed sites, while small yellow dots represent undisturbed habitats. Disturbance intensity was measured by the proportion of forest pixels lost between 2000 and 2017 in a buffer of $5 \mathrm{~km}$ radius around the supersites, using the global 30-m resolution Landsat (Hansen et al. 2013)

\section{Building a Ground-Based Earth Observation Mission}

There has been a shift over the past few decades toward freely available Earth Observation data, and NASA and ESA have adopted open data policies with the aim of accelerating science and applications (Turner et al., 2015). Earth Observation data are typically delivered free at the point of use, using technology that has cost space agencies and their funding governments hundreds of millions of dollars or euros to develop and launch. Once the sensors are installed in orbit, they continue to supply data at, relatively, limited recurrent cost. Because nations have provided the core investment, they can rightly insist that Earth Observation data are provided for free to the entire scientific community (although conditions of use may vary among space agencies).

In situ ground-based datasets stand in stark contrast with this situation, because the more reliable data are obtained by human specialists, who are paid for gathering them, verifying them, and maintaining databases over decades. In addition to data collection costs, data curation and coordination is also costly, and these costs do not come down with time. Most of the ground forest stand data available as of 2018, and summarized above, were collected and processed through long-term collaborations and with funds mobilized, typically, by individual scientists within the scientific community of many countries, rather than national efforts, and for a multitude of purposes. Few were collected with the express purpose of calibrating or validating remote imagery. It should not be assumed that the level of funding provided to these science projects will persist with the same intensity from 2019 to 2029 . The majority of the principal investigators reside in countries with limited support from national science funding, hence relying on international collaboration to sustain their activities. It is reasonable to suppose that if the substantial future ground effort proposed in this paper is to be effectively used to support remote sensing missions, then it needs to be funded to do so. 
Costs of collecting ground data are difficult to summarise given vastly differing national circumstances of operating, for example, in Costa Rica compared to the Democratic Republic of Congo. It was estimated by FOS partners that the full cost of recensusing a single 1-ha plot in high-diversity tropical forests is on the order of $15 \mathrm{k} €$ (2016 economic conditions). This reflects the entire cost from concept to delivery of the highest possible quality data with accurate tree dimensions and identification. For instance, to fully recensus 600 1-ha plots across all four tropical continents included in the ForestPlots database, the full cost would be $9 \mathrm{M} €$ per remeasurement cycle. A similar figure is to be expected for the ForestGEO network. These costs are indicative, but result from decades of experience in establishing and maintaining tropical forest plots across the world. If satellite estimates of biomass are to be of high quality and serve the widest use, these costs should be factored into the calibration and validation strategies of EO missions.

A coordinated and global ground-based monitoring of forests would benefit several sectors of science and the society and would be of direct use to biomass-related spaceborne missions. It would allow the collection and maintenance of ground-based databases for the lifetime of the currently planned missions and potentially for longer periods. In addition, this 'ground mission' would help consolidate the remote sensing/ecology nexus, helping to bridge the gap between these two scientific communities and increase the value of both ground and remotely sensed data. Finally, the study sites could become valuable beyond the currently planned biomass missions. For instance, several missions are committed to measuring photosynthetic activity through solar-induced fluorescence or aim to monitor biodiversity using hyperspectral imagery. In these situations, it is also essential to validate the concept of these missions at a set of reference sites that can be appropriately accessed, equipped, and maintained.

Acknowledgements We thank the organizers of the ISSI ESA Meeting held in Bern, Switzerland, in November 2017 for stimulating discussions and for their invitation to submit this paper. We gratefully acknowledge funding by 'Investissement d'Avenir' programs managed by Agence Nationale de la Recherche (CEBA, ref. ANR-10-LABX-25-01; TULIP, ref. ANR-10-LABX-41), from CNES and from ESA (IFBN Project 4000114425/15/NL/FF/gp, as part of the BIOMASS mission program).

\section{References}

Anderson-Teixeira KJ, Davies SJ, Bennett AC, Gonzalez-Akre EB, Muller-Landau HC, Wright SJ et al (2015) CTFS-Forest GEO: a worldwide network monitoring forests in an era of global change. Glob Change Biol 21:528-549

Ashton PS (1964) Ecological studies in the mixed dipterocarp forests of Brunei State. Clarendon Press, Oxford

Asner GP, Powell GV, Mascaro J, Knapp DE, Clark JK, Jacobson J et al (2010) High-resolution forest carbon stocks and emissions in the Amazon. Proc Natl Acad Sci 107:16738-16742

Avitabile V, Herold M, Heuvelink GB, Lewis SL, Phillips OL, Asner GP et al (2016) An integrated pantropical biomass map using multiple reference datasets. Glob Change Biol 22:1406-1420

Baccini A, Goetz SJ, Walker WS, Laporte NT, Sun M, Sulla-Menashe D, Hackler J et al (2012) Estimated carbon dioxide emissions from tropical deforestation improved by carbon-density maps. Nat Clim Change 2:182-185

Brede B, Lau A, Bartholomeus HM, Kooistra L (2017) Comparing RIEGL RiCOPTER UAV LiDAR derived canopy height and DBH with terrestrial LiDAR. Sensors 17:2371

Brown S, Lugo AE (1982) The storage and production of organic matter in tropical forests and their role in the global carbon cycle. Biotropica 14(3):161-187 
Calders K, Origo N, Burt A, Disney MI, Nightingale J, Raumonen P, Åkerblom M, Malhi Y, Lewis P (2018) Realistic forest stand reconstruction from terrestrial LiDAR for radiative transfer modelling. Remote Sens 10:933

Castilho CV, Magnusson WE, de Araújo RN, Luizao RC, Luizao FJ, Lima AP, Higuchi N (2006) Variation in aboveground tree live biomass in a central Amazonian Forest: effects of soil and topography. For Ecol Manag 234:85-96

Chapman B, Kasischke ES (2018) Evaluation of above study region sites for future calibration and validation of Nisar Science requirements. In IGARSS 2018-2018 IEEE International Geoscience and Remote Sensing Symposium, pp 8279-8281

Chave J, Réjou-Méchain M, Búrquez A, Chidumayo E, Colgan MS, Delitti WB et al (2014) Improved allometric models to estimate the aboveground biomass of tropical trees. Glob Change Biol 20:3177-3190

Chazdon RL, Broadbent EN, Rozendaal DM, Bongers F, Zambrano AMA, Aide TM et al (2016) Carbon sequestration potential of second-growth forest regeneration in the Latin American tropics. Sci Adv 2(5):e1501639

Clark DB, Clark DA (2000) Landscape-scale variation in forest structure and biomass in a tropical rain forest. For Ecol Manag 137:185-198

Clark DB, Kellner JR (2012) Tropical forest biomass estimation and the fallacy of misplaced concreteness. J Veg Sci 23:1191-1196

Condit R (1998) Tropical forest census plots: methods and results from Barro Colorado Island, Panama and a comparison with other plots. Springer, Berlin

Disney MI, Boni Vicari M, Calders K, Burt A, Lewis S, Raumonen P, Wilkes P (2018) Weighing trees with lasers: advances, challenges and opportunities. R Soc Interface Focus 8(2):20170048

Drake JB, Dubayah RO, Clark DB, Knox RG, Blair JB, Hofton MA et al (2002) Estimation of tropical forest structural characteristics using large-footprint lidar. Remote Sens Environ 79:305-319

Dubois-Fernandez PC, Le Toan T, Daniel S, Oriot H, Chave J, Blanc L et al (2012) The TropiSAR airborne campaign in French Guiana: objectives, description, and observed temporal behavior of the backscatter signal. IEEE Trans Geosci Remote Sens 50:3228-3241

Fridman J, Holm S, Nilsson M, Nilsson P, Ringvall AH, Ståhl G (2014) Adapting National Forest Inventories to changing requirements - the case of the Swedish National Forest Inventory at the turn of the 20th century. Silva Fennica 48(3):29

Frolking S, Palace MW, Clark DB, Chambers JQ, Shugart HH, Hurtt GC (2009) Forest disturbance and recovery: a general review in the context of spaceborne remote sensing of impacts on aboveground biomass and canopy structure. J Geophys Res Biogeosci 114(G2):222

Gentry AH (1988) Changes in plant community diversity and floristic composition on environmental and geographical gradients. Ann Mo Bot Gard 75(1):1-34

Golley FB (1993) A history of the ecosystem concept in ecology: more than the sum of the parts. Yale University Press, New Haven

Grassi G, House J, Dentener F, Federici S, den Elzen M, Penman J (2017) The key role of forests in meeting climate targets requires science for credible mitigation. Nat Clim Chang 7:220

Hansen MC, Potapov PV, Moore R, Hancher M, Turubanova SAA, Tyukavina A et al (2013) High resolution global maps of 21st-century forest cover change. Science 342:850-853

IPCC (2018) Special report on global warming of $1.5^{\circ} \mathrm{C}$. https ://www.ipcc.ch/sr15/

Johnson MO, Galbraith D, Gloor M, De Deurwaerder H, Guimberteau M, Rammig A et al (2016) Variation in stem mortality rates determines patterns of above-ground biomass in Amazonian forests: implications for dynamic global vegetation models. Glob Change Biol 22:3996-4013

Keeling HC, Phillips OL (2007) The global relationship between forest productivity and biomass. Glob Ecol Biogeogr 16:618-631 
Labrière N, Tao S, Chave J, Scipal K, Le Toan T, Abernethy K et al (2018) In Situ reference datasets from the TropiSAR and AfriSAR campaigns in support of upcoming spaceborne biomass missions. IEEE J Sel Top Appl Earth Obs Remote Sens 99:1-11

Lutz JA, Furniss TJ, Johnson DJ, Davies SJ, Allen D, Alonso A et al (2018) Global importance of large diameter trees. Glob Ecol Biogeogr 27(7):849-864

Le Quéré C, Andrew RM, Friedlingstein P, Sitch S, Pongratz J, Manning AC et al (2018) Global carbon budget 2017. Earth Syst Sci Data 10:405-448

Le Toan T, Quegan S, Davidson MWJ, Balzter H, Paillou P, Papathanassiou K et al (2011) The BIOMASS mission: mapping global forest biomass to better understand the terrestrial carbon cycle. Remote Sens Environ 115:2850-2860

Lewis SL, Sonké B, Sunderland T, Begne SK, Lopez-Gonzalez G, Van Der Heijden GM et al (2013) Aboveground biomass and structure of 260 African tropical forests. Philos Trans R Soc B 368:20120295

Malhi Y, Wood D, Baker TR, Wright J, Phillips OL, Cochrane T, Meir P et al (2004) The regional variation of aboveground live biomass in old-growth Amazonian forests. Glob Change Biol 12:1107-1138

Martin AR, Doraisami M, Thomas SC (2018) Global patterns in wood carbon concentration across the world's trees and forests. Nat Geosci 11(12):915

Mitchard ET, Feldpausch TR, Brienen RJ, Lopez-Gonzalez G, Monteagudo A, Baker TR et al (2014) Markedly divergent estimates of Amazon forest carbon density from ground plots and satellites. Glob Ecol Biogeogr 23:935-946

Mokany K, Raison RJ, Prokushkin AS (2006) Critical analysis of root:shoot ratios in terrestrial biomes. Glob Change Biol 12:84-96

NASA-ESA-Smithsonian Workshop on Calibration and Validation of Upcoming Satellite Missions on Forest Structure and Biomass, Washington DC, 2016. (https ://nisar .jpl.nasa.gov/files /nisar /NISAR_Veget ation Bioma ss_Works hop_Repor t.pdf)

Nogueira EM, Nelson BW, Fearnside PM (2006) Volume and biomass of trees in central Amazonia: influence of irregularly shaped and hollow trunks. For Ecol Manag 227:14-21

Pan Y, Birdsey RA, Fang J, Houghton R, Kauppi PE, Kurz WA et al (2011) A large and persistent carbon sink in the world's forests. Science 1(2):1-3. https ://doi.org/10.1126/scien ce.12016 09

Paul KI, Roxburgh SH, Chave J, England JR, Zerihun A, Specht A et al (2016) Testing the generality of above-ground biomass allometry across plant functional types at the continent scale. Glob Change Biol 22:2106-2124

Paul KI, Larmour J, Specht A, Zerihun A, Ritson P, Roxburgh SH et al (2019) Testing the generality of below-ground biomass allometry across plant functional types. For Ecol Manag 432:102-114

Phillips J, Duque A, Scott C, Wayson C, Galindo G, Cabrera E et al (2016) Live aboveground carbon stocks in natural forests of Colombia. For Ecol Manag 374:119-128

Réjou-Méchain M, Muller-Landau HC, Detto M, Thomas SC, Toan TL, Saatchi SS et al (2014) Local spatial structure of forest biomass and its consequences for remote sensing of carbon stocks. Biogeosciences 11:5711

Réjou-Méchain M, Tanguy A, Piponiot C, Chave J, Hérault B (2017) Biomass: an r package for estimating above-ground biomass and its uncertainty in tropical forests. Methods Ecol Evol 8:1163-1167

Saatchi SS, Harris NL, Brown S, Lefsky M, Mitchard ET, Salas W, Zutta BR et al (2011a) Benchmark map of forest carbon stocks in tropical regions across three continents. Proc Natl Acad Sci USA 108:9899-9904

Saatchi S, Marlier M, Chazdon RL, Clark DB, Russell AE (2011b) Impact of spatial variability of tropical forest structure on radar estimation of aboveground biomass. Remote Sens Environ 115:28362849

Schimel D, Pavlick R, Fisher JB, Asner GP, Saatchi S, Townsend P et al (2015) Observing terrestrial ecosystems and the carbon cycle from space. Glob Change Biol 21:1762-1776 
Shugart HH, Saatchi S, Hall FG (2010) Importance of structure and its measurement in quantifying function of forest ecosystems. J Geophys Res Biogeosci. https ://doi.org/10.1029/2009J G0009 93

Sillett SC, Van Pelt R, Carroll AL, Campbell-Spickler J, Coonen EJ, Iberle B (2019) Allometric equations for Sequoia sempervirens in forests of different ages. For Ecol Manag 433:349-363

Sist P, Rutishauser E, Peña-Claros M, Shenkin A, Hérault B, Blanc L et al (2015) The Tropical managed Forests Observatory: a research network addressing the future of tropical logged forests. Appl Veg Sci 18:171-174

Smith WB (2002) Forest inventory and analysis: a national inventory and monitoring program. Environ Pollut 116:S233-S242

Stegen JC, Swenson NG, Enquist BJ, White EP, Phillips OL, Jørgensen PM, Weiser MD, Mendoza AM, Vargas PN (2011) Variation in above-ground forest biomass across broad climatic gradients. Glob Ecol Biogeogr 20:744-754

Sullivan MJ, Talbot J, Lewis SL, Phillips OL, Qie L, Begne SK et al (2017) Diversity and carbon storage across the tropical forest biome. Scientific Rep 7:39102

Turner W, Rondinini C, Pettorelli N, Mora B, Leidner AK, Szantoi Z et al (2015) Free and open-access satellite data are key to biodiversity conservation. Biol Cons 182:173-176

Xu L, Saatchi SS, Shapiro A, Meyer V, Ferraz A, Yang Y et al (2017) Spatial distribution of carbon stored in forests of the Democratic Republic of Congo. Sci Rep 7:15030

\section{Affiliations}

Jérôme Chave ${ }^{1}$, Stuart J. Davies ${ }^{2}$, Oliver L. Phillips ${ }^{3}$, Simon L. Lewis ${ }^{3,8}$, Plinio Sist ${ }^{4}$, Dmitry Schepaschenko ${ }^{5}$, John Armston ${ }^{6}$, Tim R. Baker ${ }^{3}$, David Coomes ${ }^{7}$, Mathias Disney ${ }^{8,9}$, Laura Duncanson ${ }^{6}$, Bruno Hérault ${ }^{4,10}$, Nicolas Labrière ${ }^{1}$, Victoria Meyer ${ }^{11}$, Maxime Réjou-Méchain ${ }^{12}$, Klaus Scipal ${ }^{13}$, Sassan Saatchi ${ }^{11}$

${ }^{1}$ Université Toulouse 3 Paul Sabatier, CNRS, ENFA, UMR 5174 Evolution et Diversité Biologique (EDB), 31062 Toulouse, France

${ }^{2}$ Center for Tropical Forest Science-Forest Global Earth Observatory, Smithsonian Tropical Research Institute, Washington, DC 20560, USA

${ }^{3}$ School of Geography, University of Leeds, Leeds LS2 9JT, UK

${ }^{4}$ Cirad, Univ Montpellier, UR Forests \& Societies, 34398 Montpellier Cedex 5, France

${ }^{5}$ International Institute for Applied Systems Analysis, Schlossplatz 1, 2361 Laxenburg, Austria

${ }^{6}$ Department of Geographical Sciences, University of Maryland, College Park, MD 20742, USA

${ }^{7}$ Department of Plant Sciences, Forest Ecology and Conservation group, University of Cambridge, Cambridge CB2 3EA, UK

${ }^{8}$ Department of Geography, University College London, London WC1E 6BT, UK

${ }^{9}$ UKNERC National Centre for Earth Observation (NCEO), Swindon SN2 1EU, UK

${ }^{10}$ Institut National Polytechnique Félix Houphouët-Boigny, INP-HB, Yamoussoukro, Ivory Coast

${ }^{11}$ Jet Propulsion Laboratory, California Institute of Technology, Pasadena, CA 91109, USA

${ }^{12}$ AMAP, IRD, CNRS, CIRAD, INRA, Univ Montpellier, 34090 Montpellier, France

${ }^{13}$ ESA-ESTEC, 2201 AZ Noordwijk, The Netherlands 